\title{
Improving Energy Conservation in Wireless Sensor Networks using Energy Harvesting System
}

\author{
Abdul Rashid, Faheem Khan, Toor Gul, \\ Fakhr-e-Alam, and Shujaat Ali \\ Department of Computer Science \\ Bacha Khan University Charsadda, \\ Pakistan
}

\begin{abstract}
Wireless Sensor Networks assume an imperative part to monitor and gather information from complex geological ranges. Energy conservation plays a fundamental role in WSNs since such sensor networks are designed to be located in dangerous and non-accessible areas and has gained popularity since the last decade. The main issue of Wireless Sensor Network is energy consumption. Therefore, management of energy consumption of the sensor node is the main area of our research. Sensor nodes use non-changeable batteries for power supply and the lifetime of Sensor node greatly depends on these batteries. The replacement of these batteries is very difficult in many applications, such as an alternative solution to this problem is to use Energy Harvesting system in Wireless Sensor Network to provide a permanent power supply to sensor nodes. This process of extracting energies from nature and converting it into electrical energy is called energy harvesting. Energy can be harvested from the environment for sensor nodes. There are many sources of energies in nature like solar, wind and thermal which can be harvested and used for WSNs. In this research, we suggest to use energy harvesting system for Cluster Heads in a clustering based Wireless Sensor Networks. We will compare our proposed technique to a well-known clustering algorithm Low Energy Adaptive Cluster Hierarchy (LEACH).
\end{abstract}

Keywords-Wireless sensor network; Low Energy Adaptive Cluster Hierarchy (LEACH); clustering; cluster head; energy harvesting; energy conservation

\section{INTRODUCTION}

A Wireless Sensor Network (WSN) is composed of a large number of small dispersed devices known as sensor nodes that are closely deployed in the environment sense changes. The location of nodes in a sensor network may not be predetermined and usually have a unique sensor node called Base Station (BS). All member nodes will forward data to BS either directly or through multi hop transmission. A base station may be either static or dynamic sensor node and provides wireless connectivity to its users. It is usually more capable than other sensor nodes in the WSN [1], [2] (see Fig. 1).

When sensor nodes in a WSN [3] run out of energy, they stop working which causes the whole sensor network to fail. Therefore, the main issue of WSN is energy conservation. Hence, such protocols should be designed which use minimum energy and should be utilized during sensing, processing and transmission. In Wireless Sensor Network, energy consumption is a vital challenge for sensor node for sensing and transmitting the data to the closest SN or the base station. The

\author{
Samiullah Khan, \\ Fahim Khan Khalil \\ Institute of Business Management Sciences \\ The University of Agriculture Peshawar \\ Pakistan
}

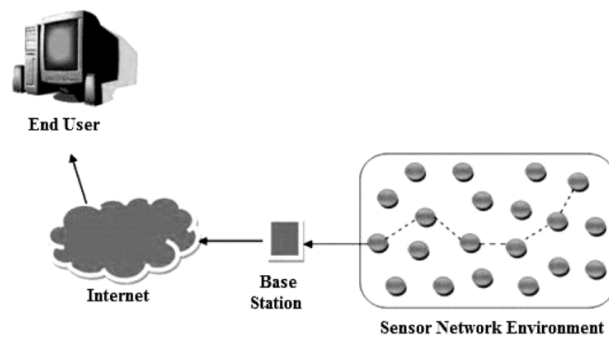

Fig. 1. Simulation results.

key requirement of a WSN is to minimize energy consumption and prolong network lifetime. Communication among sensor nodes stops when these sensor nodes lose their battery power [4].

\section{A. Challenges to Wireless Sensor Network}

Several challenges still need to be faced by WSNs. The main challenges and essential design limitation which affect the performance of Wireless Sensor Network are discussed here [5]:

1) Resource limitations: Sensing nodes are bounded by energy, data processing abilities, memory and the data transmission rate to be gained.

2) Security: The Wireless Sensor Network must be secure to control illegal entities spreading false data to the sensor node or giving wrong information to the other sensor nodes and possibly causing significant damage to the sensor nodes.

3) Self-Management: After deployment of the sensor nodes, the user has less interaction with these sensor nodes with no infrastructure support or the capability to keep and repair itself. Therefore, the sensor nodes must be self-organised in a way that must be configured, work together with other sensor nodes.

4) Heterogeneity: It is a group of sensor nodes which are not identical and do not have similar capabilities, i.e. some sensor nodes are more powerful than others. Heterogeneity arises when two different sensor networks need to communicate with each other. There will be some mechanism which is required to enable efficient information exchange among these networks. 
5) Other Challenges: Several other challenges may affect the design of Wireless Sensor Networks. For example, a group of sensor nodes is combined into a portable object, like a robot or automobile. This results in constant sensor network topologies being altered, which require repetitive changes. There is a need for routing (e.g., modifying neighbour lists), Media Access Control (e.g., modifying density), and data gathering.

\section{B. Energy Harvesting in WSN}

Energy harvesting [6] refers to collecting energy from surrounding (sun, wind) or other sources of energies (body heat, finger stroke, foot strikes) and converting these energies to electrical energy. Energies from external sources can be harvested to power the nodes to increase their lifetime. Energy harvesting (EH) has significantly improved the lifetime of a WSN and enabling new devices much more reliable.

Thanks to Energy Harvesting, the general model of getting the longest possible lifetime while still giving a good sufficient result has moved in support of providing best possible outcomes with the amount of available of energy.

Energy Harvesting Wireless Sensor Networks are normal WSNs, where additional power sources assist the primary battery or entirely replaced by them. The main idea is to retrieve the power present in the nearby surroundings of a sensor node, transform it to a usable form using appropriate types of transducers and ultimately utilise it to power the sensor node themselves. If the power source used to stimulate the sensor network have enough energy level and always available then, EH supply a network with unlimited power and an approximately infinite lifetime.

Lastly, security for Wireless Sensor Networks is the critical concern. Given the level of confidence that we place into these networks, making sure that the data that a sensor node transmits should not be accessible to outside unauthorised users, or that these similar data can be altered only by the authorised users. These are the objectives of computer security [7].

There are some energy-harvesting sensor nods which are commercially available in the market such as Crossbow MI$\mathrm{CAz}$ node with solar energy-harvesting sample circuit. Currently, available energy harvesting design for sensor nodes is classified into the following types:

\section{- Harvest-Use architecture}

\section{- Harvest-Store-Use architecture.}

In the harvest-use architecture [8], the harvesting architecture directly provides energy to the sensors. The harvest-storeuse architecture [9] consists of a unit for storing harvested energy and also powers the SNs. When the harvested energies are more than the needs of the SNs, then the extra energies are stored for future use. Secondary storage is backup storage for situations when the Primary storage is running out of energy. In day time, harvested energies are used directly as well as stored for future use. In night time, the stored power is utilized to provide energies to the SNs.

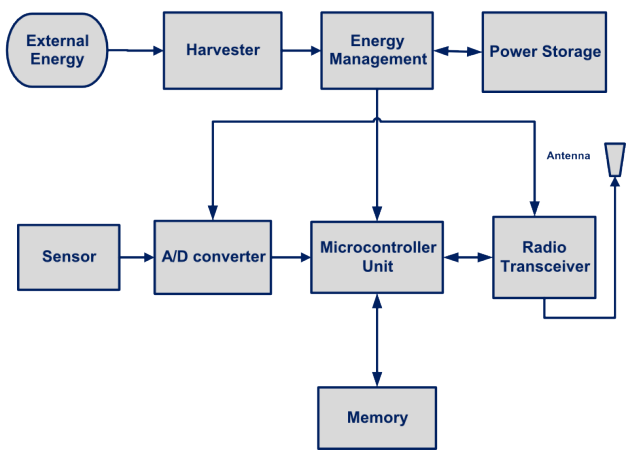

Fig. 2. System architecture of a wireless node with energy harvesters.

\section{Sensor Node Architecture with Harvesting System}

The structure of harvesting based sensor node contains the following components (see Fig. 2):

- The harvester, which converts external energy or human-created energy to electrical energy.

- An energy management unit, that gathers electrical energy from the harvester for storage or distributes it to the other components for direct use.

- Power storage, store energy for future use.

- A microcontroller unit.

- A transceiver, for receiving and transmitting information.

- Sensor.

- An Analog/Digital converter, to convert the analog signal into digital signal and provides it available to the microcontroller unit for further processing.

- Memory unit for data storage.

\section{Application of Energy Harvesting}

Several daily life applications are based on harvesting system. Energy harvesting from external sources, where a distant application is installed, and where such external energy source is unlimited, is the best alternative to expensive batteries. This is free power source available during the lifespan of the application [10].

Energy harvesting concept is used in a variety of applications in WSN. Some ad-hoc networks use minimum energy path to improve energy consumption at an SN such that the limited resources at SNs can be used more efficiently. In the meantime, if a low energy route is regularly used, this will cause decreasing in the SN energy along that route and may even cause network partition. Hence optimum routes based on energy efficient routing protocols and energy harvesting may be used to enhance the performance of the sensor network. Energy harvesting eliminates the need of battery replacement, which results to extend the lifetime of SNs.

Energy harvesting also has some industrial applications, e.g. the development of TEG using an AC Condenser, which uses measurements from thermometer which is placed in the condenser unit is the latest application [11]. The TEG had an 
energy producing capability of 20Watt. Piezoelectric shoes are a new application of energy harvesting in which piezoelectric material is implanted in the sole of the shoe. A sensor inside the shoes senses the running, walking, or other vibrations occurring on the piezoelectric material and transforms it into electrical energy which is then used to power small electronic devices. An average of $331 \mu \mathrm{W} / \mathrm{cm} 2$ was generated while walking. Another application of energy harvesting is in a club atmosphere in which energy harvesting conversion technique is placed on the dance floor in which electrical energy is produced from the dancing motion. The produced energy was utilised to provide energy to LED lighting systems in the club.

The energy harvesting also has applications in portable medical devices. These devices are likely to be small in size, lightweight and either to be wearable or inserted into the body. The dependence on batteries needs to be reduced in this field by using energy harvesting system.

Further research is going on, and more applications are introduced. These applications are now focusing on the grouping of many sources rather using a single source as can be used in case of Powerball, piezoelectric shoe and humanpowered dance floor. Here the mechanical energy in the shape of vibrations is converted into electrical energy.

\section{E. Nature of Energy Sources}

Let us discuss the nature of energy (power) availability in everyday application situations.

1) Photovoltaic energy: Whereas panels that are able of energy harvesting from internal lighting have been developed, most photovoltaic sheets are used for energy harvesting from the sunlight. The exchange efficiency and electrical properties of current photovoltaic sheets are well studied [12]. Depends upon the season, environmental location and the existence or nonexistence of shadow creating entities such as buildings and trees, solar energy follows a tendency such that it raised as the day improvement to the point of time where it is maximum and then decreases gradually. After this, a stage of no energy availability follows at night.

2) Vibration energy: Vibration energy is taking out by piezoelectric fibres that are usually made of lead zirconate titanate that shows a noticeable piezoelectric effect. Roundy and some others researchers have confirmed that these devices can extract up to $101 \mathrm{~W} / \mathrm{cm} 3$ [13]. If such an energy output were to maintained for only a one second, the sensor could monitor and forward thirty bytes of data at a transmission power of $+5 \mathrm{dBm}$ which is a usual application requirement in WSNs. Also, the difference is seen at different periods, for instance, depending on the traffic on a bridge or the movement of people in a room.

3) Mechanical energy: Mechanical energy is harvested by converting mechanical energy into electrical energy by using vibrations, pressure, and strain from high-pressure motors and force.

4) Electromagnetic energy: Electromagnetic energy is harvested by using Faradays law of electromagnetic induction. Here, an inductive spring-mass system is used for converting mechanical energy into electrical. It generates voltage by the movement of a mass of magnetic material through a magnetic eld.
5) Thermoelectric energy: Thermoelectric energy is harvested from temperature dierence (thermal gradients) using Thermoelectric Power Generators (TEGs).

6) Radio frequency energy: Radio Frequency energy harvesting is the process of converting electromagnetic energy into electrical energy. There are several Radio Frequency power sources such as television and radio broadcasting, mobile phone, Wi-Fi communications, microwaves and EM signals.

7) Wind energy: Wind energy is harvested by converting air ow (e.g., wind) energy into electricity. The wind turbine is used for linear motion coming from wind for generating electricity.

8) Biochemical energy: Biochemical energy is harvested from converting oxygen and endogenous substances into electricity through electrochemical reactions.

In the rest of the paper, Section II gives an overview about the stat of art schemes related energy harvesting systems. The proposed technique thoroughly explained in Section III. Realistic simulation scenarios and their results are discussed in Section IV. Finally, Section V gives the concluding remarks about the finding of this research article.

\section{LITERATURE REVIEW}

In this section, a portion of the topology control calculations in Energy Harvesting-Wireless Sensor Networks and also in battery-fuelled Wireless Sensor Networks are exhibited. We give an outline thought of these calculations. Energy-aware routing protocols for WSN are a very demanding research area. Due to the restrictions of wireless sensor nodes, conventional routing protocols are not appropriate for WSN. Data aggregation is a simple mechanism for decreasing the number of packets forwarded over the network because data processing for excessive aggregation amount of power as compared to transmitting multiple packets having identical data [14], [15].

LEACH is a well-known clustering based protocol [16]. In LEACH sensor nodes are organised into the cluster. Each cluster has cluster head and member nodes. Cluster heads in each cluster are selected randomly. The main disadvantage of LEACH is that if a sensor node with less residual energy is selected as cluster head would die quickly; ultimately the whole cluster would become non-functional. LEACH performs local processing to reduce the amount of data being transmitted to the BS, therefore reducing energy consumption and improving network lifetime.

In this study, a game theory-based dispersed EnergyHarvesting-Aware (EHA) algorithm is proposed [17], which represents the behaviours of sensors as a game. This effort analyses the energy expenditure rate and energy-harvesting rate of every sensor node at different times. In this approach, the high harvesting energy sensor nodes assist with the low harvesting energy sensor nodes to keep the connectivity of the sensor network. The proposed algorithm first builds a beginning topology based on the Directed Local Spanning Subgraph (DLSS) algorithm. Then every sensor node tries to and an adjacent node that covers up the remote neighbour of sensor node by adjusting the communication power stepwise.

In this approach, the authors proposed a protocol in EHWSN with a hybrid storage model. The best proportion that 
reduces outage probability is extracted, and some essential guiding principle is given [18]. The development of Carbon dioxide $\left(\mathrm{CO}^{2}\right)$ sensor nodes that are powered by artificial light was proposed in [19]. For wireless communication, these nodes use IEEE 802.11. Which is the protocol commonly used in wireless LAN. The primary objective of developing IEEE 802.11-based sensing applications is the compatibility with current networks and infrastructures. The utilisation of the body heat was proposed in [20]. Any application that uses human body heat will work well as long as the external temperature is significantly below the temperature of the standard body, i.e. $98.6^{\circ} \mathrm{F}$. When the temperature of the environment is closer to that of the human body, the capability to harvest energy reduces. However, a human being living in an air-conditioned room can use the high-temperature difference. Even throughout winter seasons at Raleigh, NC, it was observed that the ranges of temperature lie from sub-zero to $15^{\circ} \mathrm{C}$. Just moving a side of the TEG (Thermoelectric Power Generators) gives $79 \mathrm{mV}$. This is enough for starting DC-DC converters.

The idea of Radio Frequency energy and thermal energy for harvesting, transmitted through a DC-DC converter, and is used for charging the battery was presented in [21]. This approach has a high probability of functioning when environmental conditions are continuously changing, as two sources are utilized. When a TeleVision or Radio station stops broadcasting transmission, Radio Frequency energy sources are not present. Still, an existence of a temperature gradient can be harvested using a TEG.

Harvesting energy from movement and vibrations have become capable of providing power to sensor nodes. In fact, several urban environments, like highways railways, bridges and human bodies are focused on vibrations. To provide power to sensor nodes from these environmental vibrations, several researchers have built efficiently and tested many models based on piezoelectric resources [22].

In this literature, different techniques about WSN are studied. Earlier research has tried up to a certain extent to overcome the problem of energy consumption and network stability using energy efficient techniques. However, still, energy consumption and network stability is the primary challenging issue in Wireless Sensor Network. Therefore, we propose a technique of energy harvesting in clustering based Wireless Sensor Network to prolong network lifetime and network stability.

\section{Proposed TeChNiQue}

As per the literature review, a lot of energy efficient algorithms are used for prolonging network lifetime in WSN. One solution to reduce energy consumption in WSN is to use clustering technique as shown in Fig. 4. In clustering, the sensor nodes are divided into common nodes (member nodes) and some special nodes called Cluster Heads $(\mathrm{CH})$. Member nodes sense data and transmit to the cluster head. Cluster head performs data aggregation on the received data from all member nodes. The $\mathrm{CH}$ then transmit the aggregated data to base station. However, there are still problems in clustering technique, i.e. frequent re-clustering or failure of the whole cluster in the case where the energy level of the $\mathrm{CH}$ depleted.

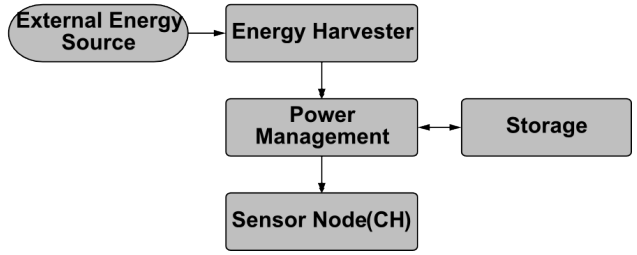

Fig. 3. Proposed architecture of cluster head with harvesting system.

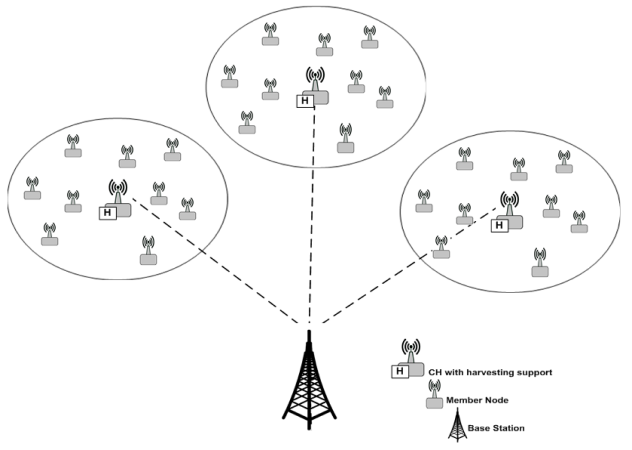

Fig. 4. Energy harvesting based clustering (EH Clustering) in WSN

So, we suggest that there should be an additional source of energy with $\mathrm{CH}$, i.e. Energy Harvesting system which provides continuous power to $\mathrm{CH}$. So, in this way, the energy of the $\mathrm{CH}$ will always be at higher level.

The proposed technique is illustrated in Fig. 3. Here energy is harvested from an external source by energy harvester and converts into electrical energy through power management unit. This energy is stored for future use or directly supplied to $\mathrm{CHs}$.

When energy harvested based sensor nodes are selected as $\mathrm{CHs}$, then $\mathrm{CH}$ broadcast an advertisement message to other sensor nodes for cluster formation. Member nodes will transmit data to these selected $\mathrm{CHs}$, CHs perform data aggregation and forward fused data to the base station as shown in Fig. 4 and 5 .

\section{A. Working Mechanism of Proposed Technique}

The proposed approach consists of the following steps:

- $\quad$ Setup phase

- Operational phase

In the setup phase, all sensor nodes will find the proper $\mathrm{CH}$ for a cluster. In the start of setup phase, the energy-harvesting nodes in the sensor network broadcast themselves as CHs. Each node determines its $\mathrm{CH}$ by measuring the strength of the received signal message and notify itself as member node by sending a join request message (Join_REQ) back to its chosen $\mathrm{CH}$ using a CSMA MAC protocol. $\mathrm{CH}$ create a TDMA schedule for data transmission coordination within the cluster and send to member nodes.

In Operational phase, sensors nodes detect events and then transmit the data to their respective $\mathrm{CHs}$ in assigned time slot defined in the TDMA schedule. $\mathrm{CH}$ performs aggregation on the received data and then transmit fused data to the Base 


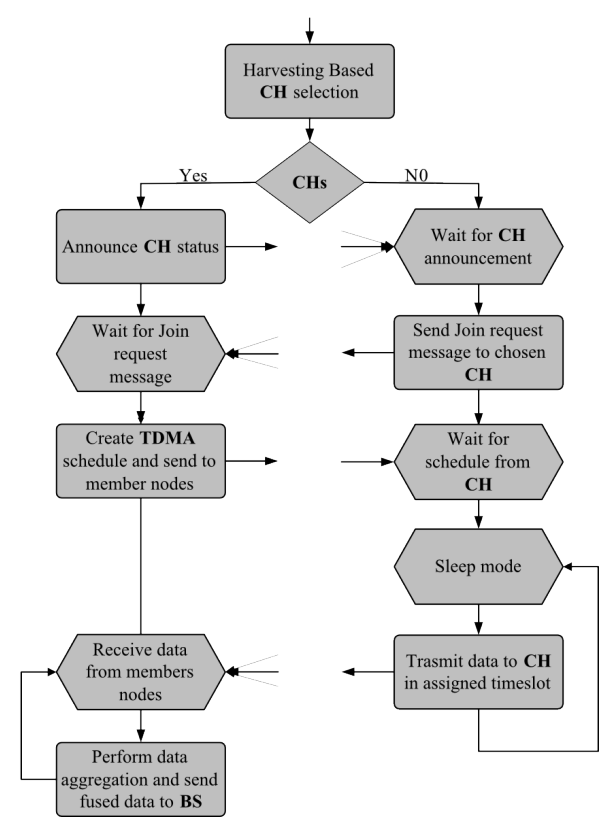

Fig. 5. Flow chart of proposed EH-Clustering technique.

Station via CSMA protocol. Thus, the $\mathrm{CH}$, battery power is utilized in two ways: (1) energy consumption while receiving data from member nodes and then performing aggregation on the data. (2) The power consumption of transmitting the aggregated data to BS.

This way, the energy consumption in common SNs will be controlled through sleep mode defined in TDMA schedule. This way the lifetime of the SNs will be prolonged.

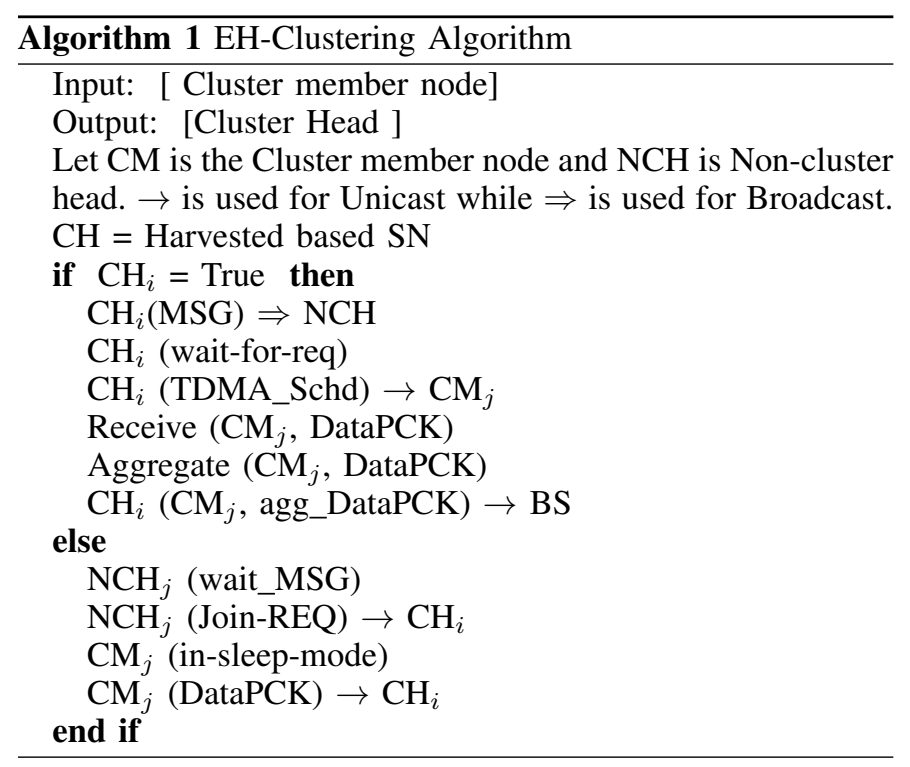

\section{RESUlTS AND DiscusSiON}

In this section, the performance of our proposed technique EH-Clustering is evaluated regarding energy consumption, throughput and lifetime of the sensor network and the results are compared with LEACH protocol. We will perform simulation on small scale as well as large-scale networks.

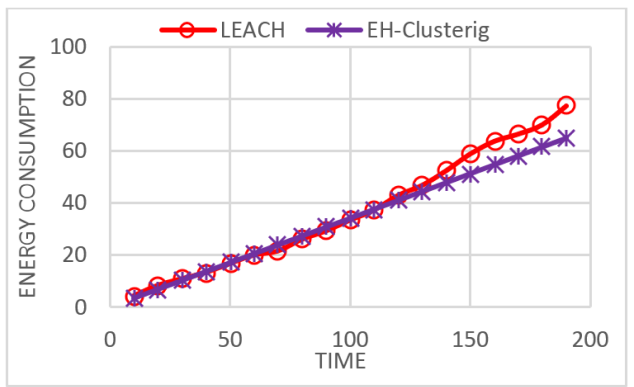

Fig. 6. Energy consumption vs Time interval for 20 nodes

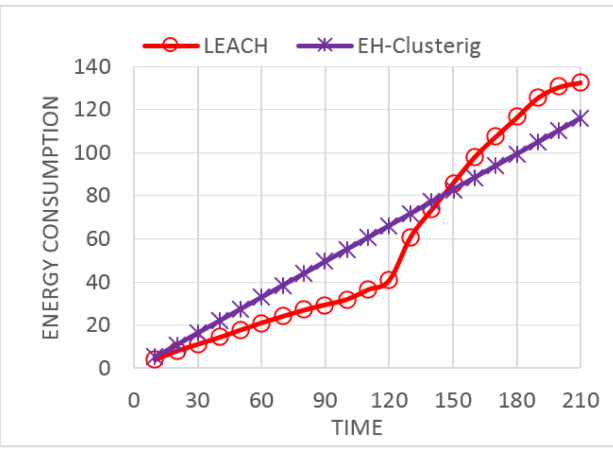

Fig. 7. Energy consumption vs time interval for 40 nodes.

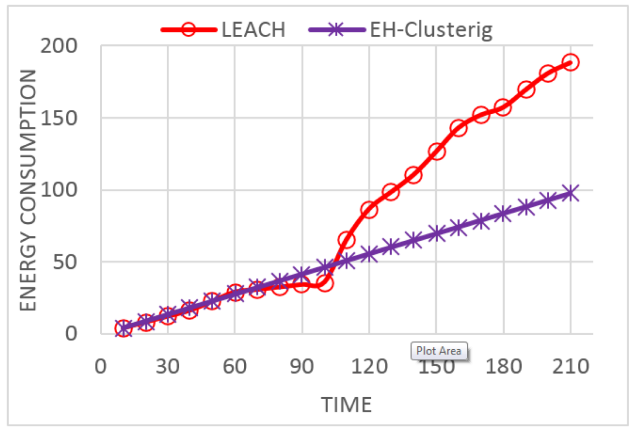

Fig. 8. Energy consumption vs Time interval for 60 nodes.

\section{A. Simulation Result Scenario for Energy Consumption}

Fig. 6 shows a comparison of energy consumption of LEACH protocol and our proposed algorithm for 20 nodes. It is clear that energy level of our proposed approach (EHClustering) is constant throughout the simulation time because regular power is supplied to $\mathrm{CH}$ from the harvesting system. The energy level of sensor nodes decreases with the passage of time in case of LEACH. So, we can say that our proposed algorithm works efficiently in this scenario.

Fig. 7 shows a comparison of energy consumption of LEACH protocol and our proposed algorithm for 40 nodes. It is clear that energy level of sensor nodes in EH-Clustering is constant throughout the simulation time because regular power is supplied to $\mathrm{CH}$ from the energy harvesting system, while the energy level of sensor nodes decreases with the passage of time in case of LEACH. So we can say that our proposed algorithm also works efficiently for 40 nodes in term of energy consumption. 


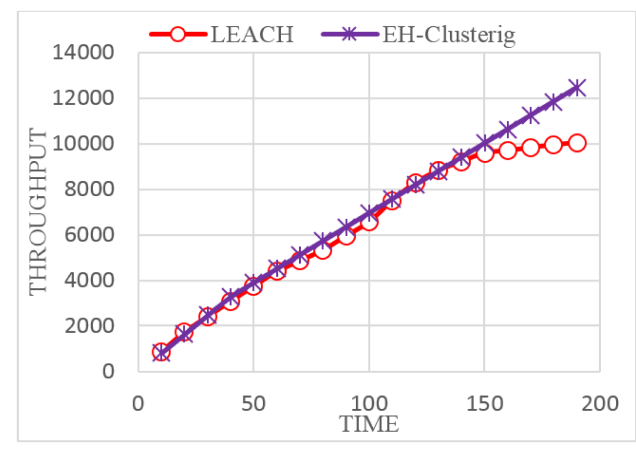

Fig. 9. Throughput vs Time interval for 20 nodes.

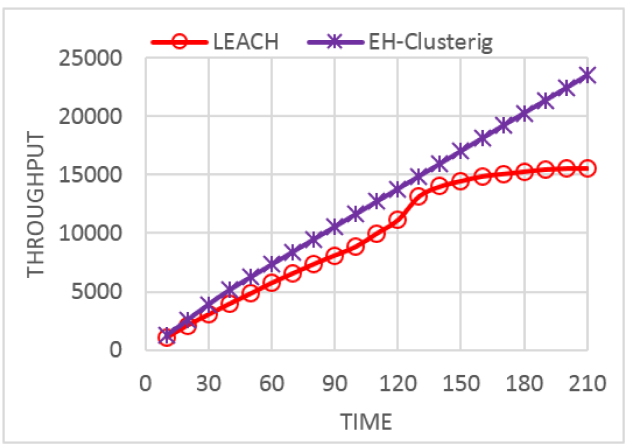

Fig. 10. Throughput vs Time interval for 40 nodes.

Fig. 8 shows a comparison of energy consumption of LEACH protocol and EH-Clustering for 60 nodes. It is clear that energy level of the whole Wireless Sensor Network in our proposed algorithm is constant throughout the simulation time, as regular power is supplied to $\mathrm{CH}$ from the energy harvesting system. The energy level of sensor nodes decreases in case of LEACH. So, we can say that our proposed algorithm also works efficiently for a network of size 60 nodes. From the above results of energy consumption for nodes 20,40 and 60 , it is clear that our proposed algorithm works efficiently for small as well as large-scale networks.

\section{B. Simulation Result Scenario of Throughput}

Fig. 9 shows the throughput comparison of LEACH and EH-Clustering in case of 20 nodes. The throughput of our proposed approach is higher as compared to LEACH for the entire simulation time because regular power is supplied to $\mathrm{CH}$ from energy harvesting system. This will result in minimum re-clustering, and the battery power of the $\mathrm{CH}$ will always be at the optimum level. As a result, the throughput will also be high. So our proposed algorithm is efficient than LEACH protocol in term of throughput for 20 nodes.

Fig. 10 shows the throughput comparison of LEACH and EH-Clustering in case of 40 nodes. From above result, it is clear that the throughput of our proposed approach is higher as compared to LEACH for the entire simulation time, as regular power is supplied to $\mathrm{CH}$ from the energy harvesting system. This will result in minimum re-clustering, and the battery power of the $\mathrm{CH}$ will always be at the optimum level. As a result, the throughput will also be high. So, our proposed algorithm also works efficiently in term of throughput for 40

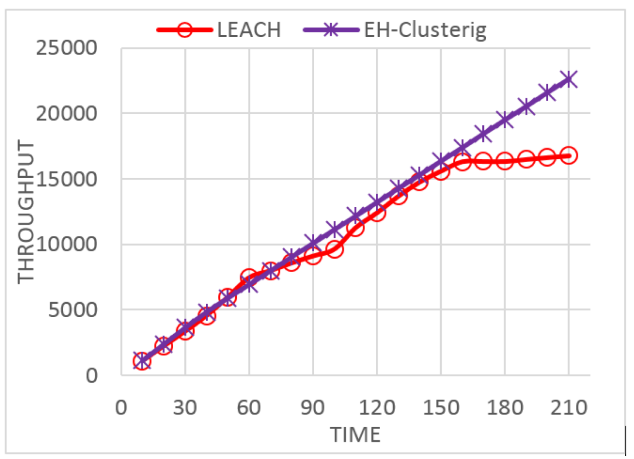

Fig. 11. Throughput vs time interval for 60 nodes.

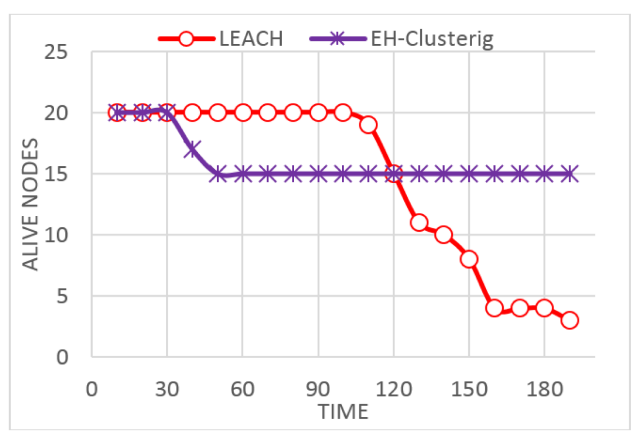

Fig. 12. Alive nodes vs Time for 20 nodes.

nodes than LEACH protocol.

Fig. 11 shows the throughput comparison of LEACH and EH-Clustering in case of 60 nodes. From above result, it is clear that the throughput of our proposed algorithm is higher as compared to LEACH for the entire simulation time, as regular power is supplied to $\mathrm{CH}$ from the energy harvesting system. This will result in minimum re-clustering, and the battery power of the $\mathrm{CH}$ will always be at the optimum level. As a result, the throughput will also be high. Hence our proposed algorithm also works efficiently in term of throughput for 60 nodes than LEACH protocol. From the above results of throughput comparisons of EH-Clustering and LEACH, we conclude that our proposed algorithm gives an efficient result for the small and large-scale network in term of throughput.

\section{Simulation Result Scenario of Alive Nodes}

Fig. 12 shows a comparison of LEACH and EH-Clustering in term of Alive nodes for 20 nodes. The alive nodes in LEACH decrease as time passes because of frequent reclustering, whereas in case of our proposed approach (EHClustering), the alive nodes are higher throughout the entire simulation time, as regular power is supplied to the $\mathrm{CH}$ from energy harvesting system, this will prolong network lifetime. So, we can say that our proposed algorithm gives the efficient result as compared to LEACH in term of Alive nodes for a network of 20 nodes.

Fig. 13 shows a comparison of LEACH and EH-Clustering in term of Alive nodes for 40 nodes. The alive nodes in LEACH decrease as time passes because of frequent reclustering, whereas in case of our proposed approach(EHClustering), the alive nodes are higher throughout the entire 


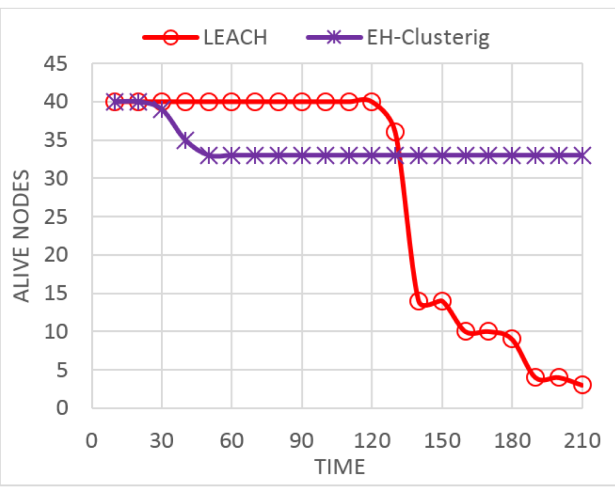

Fig. 13. Alive nodes vs Time interval for 40 nodes.

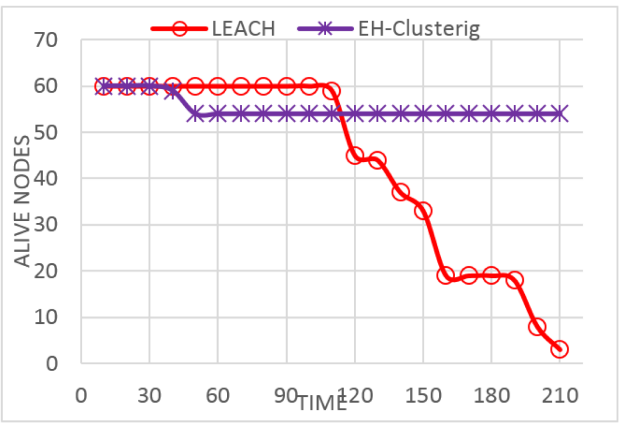

Fig. 14. Alive nodes vs Time interval for 60 nodes.

simulation time, as regular power is supplied to the $\mathrm{CH}$ from the energy harvesting system, this will prolong network lifetime. So, we can say that the lifetime of the sensor network in case of our proposed algorithm is also higher than LEACH in term of alive nodes for 40 nodes.

Fig. 14 shows a comparison of energy consumption of LEACH protocol and EH-Clustering for 60 nodes. It is clear that energy level of the whole WSN in our proposed algorithm is constant throughout the simulation time, as regular power is supplied to $\mathrm{CH}$ from the energy harvesting system. The energy level of sensor nodes decreases in case of LEACH. So, we can say that our proposed algorithm also works efficiently for a network of size 60 nodes. From the above results of energy consumption, it is clear that our proposed algorithm works efficiently for small as well as large-scale networks.

\section{COnClusion}

In this research, energy harvesting based clustering approach is proposed. Our simulation results have shown that by using energy harvesting in clustering based WSNs, the lifetime of a WSN significantly enhanced. From this research, it is clear that energy harvesting is a best alternate source of energy for Wireless Sensor Networks. Using energy-aware clustering concepts, the battery usage and computation overhead will be decreased. Energy harvesting based clustering approach enhance the energy conservation of the sensor nodes. The energy harvesting based clustering approach enhances the performance and lifetime of Wireless Sensor Networks as compared to other algorithms. The deployment of low-cost energy harvesting based sensors nodes is the main cause for the reliable communication and prolonging lifetime in Wireless Sensor Network.

\section{FUTURE RECOMMENDATION}

For future work, it is recommended to improve security in Wireless Sensor Network by using authenticated protocols to handle the malicious sensor node attack along with energy harvesting to get more robust systems.

\section{REFERENCES}

[1] A. Rathee, R. Singh and A. Nandini, Wireless Sensor Network- Challenges and Possibilities, International Journal of Computer Applications, vol. 140, no. 2, pp. 1-15, 2016.

[2] S. Zin, N. B. Anuar, M. L. Kiah and A.-S. Pathan, Routing protocol design for secure WSN : Review and open research issues, Journal of Network and Computer Applications, February 2014.

[3] S. Kumar, B. Prabhu, Rajkumar and D. S. Sophia, A Methodology For Reducing Energy Utilization In Dense Wireless Sensor Networks, International Journal of Research Granthaalayah, vol. 4, no. 1, pp. 125-130., 2016.

[4] K. Vijeta, D. Kavita and D. B. Jangra, Extended LEACH-Based Clustering Routing Protocols For WSN: A Survey, International Journal of Engineering Development and Research, vol. 5, no. 1, pp. 362-367, 2017.

[5] D. N. A. Shiltagh and A. H. Wheeb, Priority Based Transmission Rate Control with Neural Network Controller in WMSNs, Journal of Engineering, vol. 20, pp. 66-81, 2014.

[6] S. Akbari, Energy Harvesting for Wireless Sensor Networks Review, in Federated Conference on Computer Science and Information Systems, pp. 987992, 2014.

[7] A. D. Mauro and N. Dragoni, On the Impact of Energy Harvesting on Wireless Sensor Network Security, Technical University of Denmark (DTU), (DTU Compute PHD-2014; No. 349), 2015.

[8] C. Anbarasan, B. Anantharaj and N. Balaji, Cooperative Energy Allocation for Sensing and Transmission in Rechargeable WSN, International Research Journal of Latest Trends in Engineering and Technology, vol. 3, no. 1, pp. 9-18, 2016

[9] M. Singh and T. Singh, Energy Efficient, Distributed Clustering Approach for Ad Hoc Wireless Sensor Network, International Journal of Science and Research, vol. 6, no. 4, pp. 2415-2421, 2017.

[10] S. Sojan and D. R. Kulkarni, A Comprehensive Review of Energy Harvesting Techniques and its Potential Applications, International Journal of Computer Applications (0975 8887), vol. 139, no. 3, pp. 14-19, April 2016.

[11] D. F. Yildiz and K. L. Coogler, Low Power Energy Harvesting with a Thermoelectric Generator through an Air Conditioning Condenser in 121st ASEE Annual Conference \& Exposition, Indianapolis, 2014.

[12] M. A. Green, K. Emery, Y. Hishikawa, Warta and E. D. Dunlop, Solar cell efficiency tables (version 39), Progress in Photovoltaics: Research and Applications, vol. 20, no. 1, p. 1220, 2012.

[13] S. Roundy, D. Steingart, L. Frechette, P. Wright and J. Rabaey, Power sources for wireless sensor networks, Springer Berlin Heidelberg, vol. 29, no. 20, pp. 1-17.2004

[14] Anuj, Wireless Sensor Network: A Review On Data Aggregation International Journal of Innovations in Applied Sciences \& Engineering, ISSN: 2454-9258, vol. 2, pp. 11-17, 2016.

[15] Faheem khan, Sohail abbas, Samiullah khan, An Efficient and Reliable Core-Assisted Multicast Routing Protocol in Mobile Ad-Hoc Network, International Journal of Advanced Computer Science and Applications, vol7:5, 2016

[16] M. J. Usman, Z. Xing, H. Chiroma, A. Y. U. Gital, A. I. Abubakar, A. M. Usman and Herawan, Modified Low-Energy Adaptive Clustering Hierarchy Protocol for Efficient Energy Consumption in Wireless Sensor Networks for Healthcare Applications, International Review on Computers and Software, vol. 9, no. 11, pp. 1904-1915, 2014.

[17] T. Qian, A. Wei, Y. Han, Y. Liu and S. C, Energy Harvesting Aware Topology Control with Power Adaptation in Wireless Sensor Networks, Ad Hoc Network, vol. 27, no. C, pp. 44-56, April 2015. 
[18] S. Luo, R. Zhang and T. J. Lim, Optimal save-then-transmit protocol forenergy harvesting wireless transmitters, IEEE Transactions on Wireless Com-munications, vol. 12, no. 3, p. 11961207, Mar. 2013.

[19] X. Fafoutisa, T. Sorensenb and J. Madsena, Energy Harvesting Wireless Sensor Networks for Indoors Applications using IEEE 802.11, Procedia Computer Science, vol. 32 , p. 991 996, 2014.

[20] L. C. Mateu, N. P. Lucas and P. Spies, Human Body Energy Harvesting Thermogenerator for Sensing Applications, International Conference on
Sensor Technologies and Applications, p. 366372, Oct. 2007.

[21] D. Har, S. Min and T. M. Mladenov, Radio frequency energy harvesting for wireless sensor networks, International Journal of Distributed Sensor Networks, vol. 13, no. 6, 2017.

[22] A. Almusallam, R. N. Torah, D. Zhu, M. J. Tudor and S. P. Beeby, Screen-printed piezoelectric shoe-insole energy harvester using an improved flexible PZT-polymer composites, Journal of Physics: Conference, Vols. 476 (1742-6596/476/1/012108), 2013. 